\title{
Some proposed fixed target experiments with the LHC beams
}

\author{
Nataliya Topilskaya ${ }^{1, *}$ and Alexey Kurepin ${ }^{1, * *}$ \\ for the AFTER@LHC study group \\ http://after.in2p3.fr/after/index.php/Current_author_list \\ ${ }^{1}$ Institute for Nuclear Research, RAS, Moscow, Russia
}

\begin{abstract}
The physics opportunities offered by using the multi-TeV LHC beams for a fixed target experiment have been widely discussed in recent years. This mode is convenient to investigate rare processes of particle production and polarization phenomena because the expected luminosity exceeds the luminosity of the collider. The main physical goals of these experiments are: i) investigations of the large- $x$ gluon, antiquark and heavy quark content in the nucleon and nucleus; ii) investigations of the dynamics and spin of quarks and gluons inside nucleus; iii) studies of the ion-ion collisions between SPS and RHIC energies towards large rapidities. With the LHC lead beam energy scan on a fixed target it would be possible to investigate the energy range up to $72 \mathrm{GeV}$ to search for the critical point for the phase transition to the Quark Gluon Plasma (QGP).
\end{abstract}

\section{Introduction}

Physics opportunities offered by using the multi-TeV proton and ion beams at the LHC for a fixed target experiment and the corresponding physics program for heavy-ion, hadron, spin and astroparticle physics were discussed in several publications, for example [1-5]. Using this mode, referred to us as the AFTER@LHC project (A Fixed Target ExpeRiment) in the following, has a number of advantages for investigating rare processes of particle production and polarization phenomena over a broad rapidity range and using several target types, as compared to experiments at colliders.

The centre-of-mass particle energy reaches values only half as much as at the RHIC collider, but the high expected luminosity considerably increases the statistical precision of the data. For $\mathrm{p}-\mathrm{p}$ and $\mathrm{p}$-A collisions with the $7 \mathrm{TeV}$ proton beam the energy in the centre-of-mass is $\sqrt{s_{\mathrm{NN}}}=114.6 \mathrm{GeV}$ with a rapidity shift of 4.8 units $\left(y_{c . m .}=0 \rightarrow y_{\text {lab }}=4.8\right)$. For lead ions with a beam energy of $2.76 \mathrm{TeV}$ per nucleon the centre-of-mass $\mathrm{N}-\mathrm{N}$ energy is $\sqrt{s_{\mathrm{NN}}}$ $=71.8 \mathrm{GeV}$ with a rapidity shift of 4.2 units. By using the LHC lead beams from injection to the top energy in a fixed target experiment, the data will be obtained in the energy range up to $72 \mathrm{GeV}$, which could be more promising than at ultrahigh energies to search for the phase transition and determine the critical point. Indeed, at high temperature and large energy density the existence of the Quark-Gluon Plasma (QGP) is predicted by lattice Quantum

\footnotetext{
*e-mail: topilska@inr.ru

**e-mail: kurepin@inr.ru
} 
ChromoDynamics (QCD). Progress in our understanding of the nuclear matter properties requires systematic and high statistic measurements also in the low energy region. Unfortunately, the luminosity collected during the beam energy scan at RHIC decreases at lower energy. Using the high intensity LHC beams will provide measurements with significantly better statistical accuracy than at RHIC.

The fixed target experiments at the LHC with standard detectors, thanks to the rapidity shift, give an opportunity to access the high- $x$ domain by measuring the far backward region in the center of mass system. It would be possible to study p-A and A-A collisions with different nuclear targets and use polarized targets. An extended number of species for the target, including deuteron and ${ }^{3} \mathrm{He}$, allows one to carry out unique neutron studies. The target polarization gives opportunities for single spin asymmetry (SSA) measurements, which have been the object of a growing attention in the recent years at RHIC, CERN and Fermilab. At present, many accelerators have a program of studies with a fixed target (Tevatron, HERA, SPS, RHIC, NICA).

\section{Motivations}

\subsection{The high- $x$ frontier}

The AFTER@LHC project could reach the backward rapidity region corresponding to high Bjorken- $x$ partons probed in the target, and is, probably, the best program for this physics with hadron beams. At the interface between the proton and nuclear cases, the deuteron and ${ }^{3} \mathrm{He}$ have a particular place. Whereas precise measurements of the partonic structure of nucleons and nuclei at small momentum fractions $x$ are usually a strong motivation for new experimental facilities, such as the Electron-Ion Collider (EIC) or Large Hadron electron Collider ( $\mathrm{LHeC})$ projects, the structure of nucleon and nucleus at high- $x$ is almost unknown. In the high- $x$ program, the aim is to improve our understanding of the high- $x$ gluon, antiquark and heavy quark content in the nucleon and nucleus.

Thanks to the high luminosity, AFTER@LHC is expected to perform Drell-Yan (DY) measurements, $\mathrm{W}$ boson production measurements and a complete set of open heavy flavour studies. In p-p collisions, a modification of the $\mathrm{D}^{0}$-meson yield can be induced by the presence of a non-perturbative intrinsic charm (IC) component in the proton. The contribution from IC in the proton could have a non negligible impact on the neutrino production from charm fluxes at high energy. AFTER@LHC, covering the large negative Feynman- $x_{\mathrm{F}}$ region down to very low $p_{\mathrm{T}}$ for charmed hadrons is convenient to put restrictions on the IC component in the proton and therefore to high energy neutrino physics.

\subsection{The nucleon spin}

In the Spin physics program the goal is to advance in understanding the dynamics and spin of quarks and gluons inside (un)polarized nucleons. There are two types of contributions to the nucleon spin-1/2 from quarks and gluons: their spin and their Orbital Angular Momentum (OAM) $[6,7]$. Recent experimental data have shown that quarks and antiquarks account for only about $25 \%$ of proton total longitudinal spin [8], and the gluon contribution could be as large as $40 \%[9,10]$, so a significant part of the proton spin arises from the transverse dynamics of quarks and gluons, which has, however, not yet been measured. Information on the orbital motion of the partons bound inside hadrons could be obtained via Single Spin Asymmetries (SSA) measurements in different hard-scattering processes, in particular, with transversely polarized hadrons $[11,12]$. In these STSAs (denoted $A_{N}$ ), one can access leftright asymmetries in the parton distributions with respect to the plane formed by the proton 
momentum and spin directions. These asymmetries, also called the Sivers effect [13-15], are naturally related to the transverse motion of the partons inside the polarized nucleons.

AFTER@LHC can measure heavy-flavour and quarkonium production, whose STSAs are almost unknown. This includes pseudo-scalar quarkonium, quarkonium-pair and other associated production of colorless particles, and, of course, the DY process. Having its high luminosity, a highly polarized target and an access towards the large momentum fraction in the target, AFTER@LHC is probably the best set-up to carry out an inclusive set of $A_{N}$ measurements. Almost nothing is known from the experimental side about the gluon Sivers effect [16]. The polarization of not only hydrogen but also deuterium and helium-3 targets allows AFTER@LHC to probe the parton transverse dynamics in the gluon sector.

The PHENIX collaboration was the first to investigate STSAs of gluon sensitive probes in $\mathrm{J} / \psi$ production. The value of $A_{N}$ was found to be compatible with zero but with large errors, allowing for the values as large as 20\%. In the AFTER@LHC experiment it would be possible to measure STSAs for bottomonia, spin-singlet states such as $\eta_{c}$, to investigate the gluon Sivers effect and the importance of the gluon orbital angular momentum to solve the nucleon-spin problem.

\subsection{The heavy-ion physics}

Finally, the AFTER@LHC physics program concerns the study of heavy-ion collisions toward large rapidity. One of the main goals of heavy-ion (HI) physics is the search and characterization of a new state of matter, the Quark-Gluon Plasma (QGP). In this state, in which the Universe was located a few microseconds after the Big Bang, quarks and gluons moved almost freely. The existence of the QGP is a natural consequence of the QCD properties. This new state is expected to be reached when the surrounding hadronic matter is extremely compressed or heated, resulting in high momentum transfers in the system. These conditions can be achieved in ultra-relativistic collisions of nuclei and the resulting new phase can be observed using specific probes. These probes are essentially of three kinds, namely radiated particles from the plasma itself (i.e. photons), the production and suppression of heavy-quark bound states and the momentum-spectrum modification of particles.

AFTER@LHC with a N-N centre-of-mass energy of $72 \mathrm{GeV}$ provides a complementary coverage to the RHIC- and SPS-based experiments in the region of high temperatures and low baryon-chemical potentials. In Pb-A collisions, at $\sqrt{s_{\mathrm{NN}}}=72 \mathrm{GeV}$, AFTER@LHC is probing the region of high temperature (near $1.5 \mathrm{~T}_{c}$ ) and low baryon chemical potential, where the QGP formation is expected to occur. At this temperature the quarkonium excited states are expected to melt in the QGP [17], thus allowing for the calibration of its temperature. Measurements with different target types of various quarkonium states (together with open heavy flavours (HF)) as a function of the rapidity and system size would permit to scan the phase transition region. Moreover, AFTER@LHC can study the transport properties of the QGP accounting for its longitudinal expansion. Indeed, by measuring the rapidity dependence of particle yields and flow coefficients $v_{N}$, AFTER@LHC can constraint the temperature dependence of the medium shear viscosity. Proper interpretation of the A-A data, would be accompanied by a complete set of $v_{N}$ coefficient measurements in smaller systems (p-p, p-A) to study collectivity with new observables, like heavy flavour hadrons, which will be abundantly produced at AFTER@LHC. Other items of interest also include the study of heavy quarks energy loss mechanisms in the QGP via D-meson measurements as a function of rapidity and $p_{\mathrm{T}}$ (nuclear modification factor, flow, ...), and test of the factorization of initial state effects from p-A to A-A collisions with the Drell-Yan probe.

Model calculations indicate that the baryonic chemical potential $\mu_{B}$ and the temperature $\mathrm{T}$ depend on the rapidity [18-20]. Measurements conducted as a function of $y_{c . m}$. will give ac- 
cess to different $\mu_{B}$ and T values, thus, AFTER@LHC could study both the QGP regime and the expected phase transition to the hadronic gas using also different colliding systems and collecting high luminosities. Another advantage is to investigate the nucleus-target region, namely $y_{l a b}$ or $\eta_{l a b}$ close to 0 .

Quarkonia are important probes to study the deconfinement at ultra-relativistic heavyion collisions. But the mechanism of quarkonium production and suppression is not well understood in some energy and kinematical domain, where cold nuclear matter effects are expected to be large. The high statistics data give an opportunity to understand the production mechanism and estimate the contribution of cold nuclear matter effects (CNM). It would be possible to investigate not only the production of $\mathrm{J} / \psi, \psi(2 \mathrm{~S})$ and $\Upsilon(\mathrm{nS})$ mesons, but also $\eta_{c}$ and $\chi_{c}$ states. These measurements require high statistics, large acceptance and good efficiency. D-meson production will complement the charmonium one to understand heavy quark production. AFTER @LHC can also measure Drell-Yan-pair production, which is free from the final state QCD matter effects, in the 4-8 GeV mass range. Drell-Yan pair production from simple $\mathrm{p}-\mathrm{p}$ collisions to asymmetric A-B collisions along with A-p and $\mathrm{p}-\mathrm{B}$ collisions with a single experimental apparatus will give information about the initial stages of nucleus-nucleus collisions [21]. Measurements at higher energies (nominal RHIC or LHC) are extremely difficult because of the fast growing size of the combinatorial background.

\section{Technical implementations for fixed target experiments}

There are several technical options to use the high intensity LHC beams for the fixed target experiment AFTER@LHC. The target in the form of thin wire or foil ring could be placed around the main orbit of the LHC. The life time of the beam is determined by the beambeam and beam-gas interactions. After some time the particles will leave the main orbit and interact with the target. So for the fixed target measurements only halo of the beam will be used $[1,22]$.

The second option is the installation of an internal gas target in the full beam flux like SMOG at LHCb [23], storage cell target (such as HERMES target) [24] or gas-jet target (like RHIC polarimeter) [25]. The internal gas system SMOG was initially designed by LHCb Collaboration as a luminosity monitor. It works at low gas density during an extended period of time without any influence on the beam lifetime. During 2015-2017 years LHCb successfully measured $\mathrm{p}-\mathrm{Ne}, \mathrm{p}-\mathrm{He}, \mathrm{p}$-Ar collisions at $\sqrt{s_{\mathrm{NN}}}=110.4 \mathrm{GeV}$ and $\mathrm{p}-\mathrm{Ar}$ and $\mathrm{Pb}-\mathrm{Ar}$ collisions with $\sqrt{s_{\mathrm{NN}}}=68.6 \mathrm{GeV}$ for proton beam and $54.5 \mathrm{GeV}$ for the lead beam. Then p-He collisions were measured at $\sqrt{s_{\mathrm{NN}}}=110.4 \mathrm{GeV}$ and $86.6 \mathrm{GeV}$, and also p-Ne collisions at $\sqrt{s_{\mathrm{NN}}}=68.6 \mathrm{GeV}$ and $110.4 \mathrm{GeV}$. No distortion of the LHC performance was observed. Using a target such as the one of the HERMES experiment at HERA or the gas-jet target, a higher gas pressure could be obtained with an opportunity to polarize the gas target. Different systems for target polarization could be used. In general, $\mathrm{H}$ or D gas targets provide a good opportunity to reach high value of polarization. Using a target similar to HERMES system or simply a gas-jet target, the effective polarization of $\mathrm{H}$ or D can reach 0.8 [26].

The third option proposes to use a bent crystal. It can be placed near the beam in order to deflect halo particles and guide them onto an external target. The extracted beam can provide a new beamline with a solid or gaseous target and special installation for possible polarization of the gaseous target. Another opportunity relies on using a bent crystal directly in the beam halo inside the beam pipe of an existing LHC experiment. This technique is not expected to affect the LHC performance as it will use only the beam halo. Similar technique is investigated and currently used by the UA9 Collaboration as a way to improve the LHC beam collimation. Technical implementations of two options (HERMES-like target and bent crys- 
tal with an internal solid target) are currently discussed within the Physics Beyond Collider working group at CERN.

\section{Detector requirements}

The ALICE and LHCb detectors provide opportunity to be used for the fixed target program of AFTER@LHC. The LHCb detector is fully instrumented in the forward region, with excellent particle identification (PID) performances. It has a mid- to backward rapidity coverage in the center-of-mass frame in the fixed target mode, allowing one to reach large negative Feynman- $x_{\mathrm{F}}$. The ALICE detector also provides a similar coverage in the mid- to backward rapidity region thanks to its current muon arm. The long absorber upstream the muon tracking stations is a key feature to reduce the combinatorial background for Drell-Yan studies in p-A and A-A collisions. In addition, the central barrel of ALICE provides a very backward rapidity coverage in a fixed target mode with good PID capabilities, allowing to reach the end of the phase space for several identified soft probes.

The maximum instantaneous luminosities can be obtained with a HERMES-like target and the values are: $3 \times 10^{28} \mathrm{~cm}^{-2} \mathrm{~s}^{-1}$ for $\mathrm{Pb}$-Xe collisions, $2 \times 10^{32} \mathrm{~cm}^{-2} \mathrm{~s}^{-1}$ for $\mathrm{p}$-Xe collisions and $6 \times 10^{33} \mathrm{~cm}^{-2} \mathrm{~s}^{-1}$ for $\mathrm{p}-\mathrm{H}_{2}$ collisions. In the case of the heaviest nuclear collisions currently foreseen, $\mathrm{Pb}-\mathrm{Xe}$ collisions at $\sqrt{s_{\mathrm{NN}}}=72 \mathrm{GeV}$, the average number of charged particles is maximal at $\eta_{l a b}=5$ and amounts to about 600 for the most $10 \%$ central collisions according to EPOS [27, 28]. The charged particles multiplicity is shown in Fig. 1 for various heavy-ion systems as a function of the pseudorapidity in the laboratory frame and is compared to the one obtained at the LHC in a collider mode for a comparison [5].

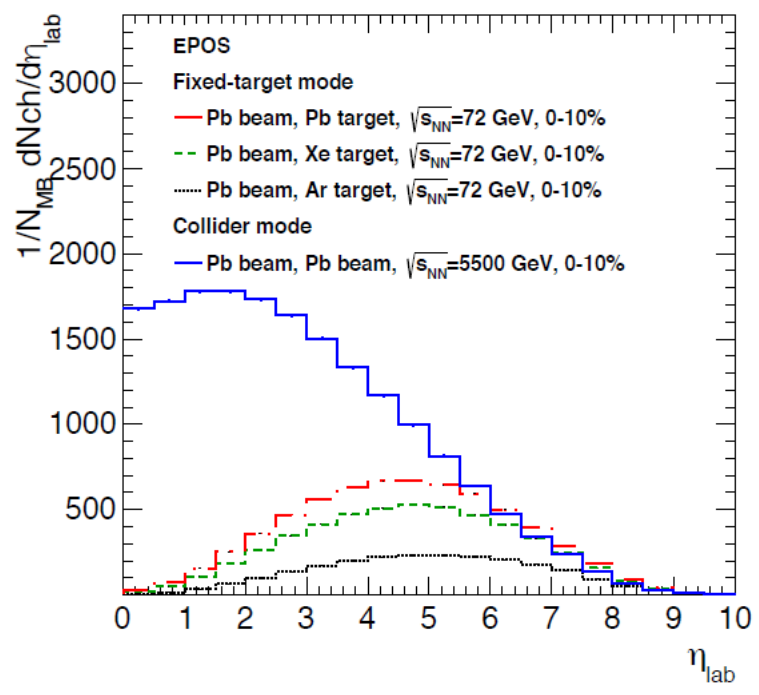

Figure 1. Averaged charged particle multiplicity as a function of the pseudo-rapidity in the laboratory frame for various heavy-ion systems

In a fixed-target mode, the multiplicity does not exceed the one obtained in $\mathrm{Pb}-\mathrm{Pb}$ collisions at $\sqrt{s_{\mathrm{NN}}}=5.5 \mathrm{TeV}$ in the collider mode. If one considers the instantaneous luminosities quoted above and the inelastic cross-sections from EPOS, one ends up with inelastic 
rates corresponding to $240 \mathrm{MHz}, 258 \mathrm{MHz}$ and $186 \mathrm{kHz}$ in $\mathrm{p}-\mathrm{p}, \mathrm{p}-\mathrm{Xe}$ and $\mathrm{Pb}-\mathrm{Xe}$ collisions, respectively. These numbers already exceed what ALICE detector (in all cases) and LHCb detector (in p-p and p-A cases) can cope with for the Runs 3 and 4. The gas density has to be leveled not to exceed $50 \mathrm{kHz}$ and $1 \mathrm{MHz}$ inelastic rates with the lead and proton beams, respectively, for ALICE and $40 \mathrm{MHz}$ with the proton beam for LHCb. This corresponds to luminosities $10^{33} \mathrm{~cm}^{-2} \mathrm{~s}^{-1}$ for $\mathrm{p}-\mathrm{H}_{2}$ collisions and $3.1 \times 10^{31} \mathrm{~cm}^{-2} \mathrm{~s}^{-1}$ for $\mathrm{p}$-Xe collisions, for the $\mathrm{LHCb}$ case. For $\mathrm{Pb}-\mathrm{Xe}$ collisions $\mathrm{LHCb}$ should be able to cope with $186 \mathrm{kHz}$ inelastic rates in principle [14]. The studies for the technical implementations are ongoing in the AFTER@LHC working group.

\section{Conclusions}

In this proceeding we have discussed the physics opportunities of using multi-TeV proton and ion beams at the LHC for a fixed target experiment and a feasibility of physics program for heavy-ion, hadron, spin and astrophysics. The fixed target experiments at the LHC with standard detectors, thanks to the rapidity shift, give an opportunity to access the high negative $x_{\mathrm{F}}$-Feinman domain by measuring the far backward rapidity region in the center-of-mass system. It would be possible to study p-p, p-A and A-A collisions with different nuclear targets and use polarized targets. An extended number of species for the target, including deuteron and ${ }^{3} \mathrm{He}$, allows one to carry out unique neutron studies. The target polarization gives opportunities for single spin asymmetry (SSA) measurements. Several possible implementations of a fixed target setup inside the existing LHC experiment (ALICE or LHCb) are currently explored. By using the LHC beams in a fixed target mode, the data could be obtained in the energy range up to $72 \mathrm{GeV}$ to search for the phase transition effects to the QGP state and determine the critical point. 15007

Acknowledgements. The work was supported in part by the RFBR/CNRS grant 18-52-

\section{References}

[1] A. B. Kurepin, N. S. Topilskaya and M. B. Golubeva, Phys. Atom. Nucl. 74, 446 (2011)

[2] S. J. Brodsky, F. Fleuret, C. Hadjidakis, J. P. Lansberg, Phys. Rept. 522, 239 (2013)

[3] J. P. Lansberg et al., Special Issue in Adv. High Energy Phys. 2015, (2015)

[4] J. P. Lansberg et al., PoS PSTP 2015, 042 (2016)

[5] C. Hadjidakis et al., arXiv:1807.00603 [hep-ex]

[6] E. Leader and C. Lorce, Phys. Rept. 541, 163 (2015)

[7] M. Wakamatsu, Int. J. Mod. Phys. A 29, 143012 (2014)

[8] D. de Florian et al., Phys. Rev. Lett. 101, 072001 (2008)

[9] I. Adamczyk et al. (STAR Collaboration), Phys. Rev. Lett. 115, 092002 (2015)

[10] D. de Florian et al., Phys. Rev. Lett. 113, 012001 (2014)

[11] U. Barone, F. Brandamante, and A. Martin, Prog. Part. Nucl. Phys. 65, 267 (2010)

[12] J. P. Lansberg et al., EJP Web Conf. 85, 02038 (2015)

[13] D. W. Sivers, Phys. Rev. D 41, 83 (1990)

[14] L. Massacrier et al., Adv. High Energy Phys. 2015, 986348 (2015)

[15] J. P. Lansberg et al., PoS DIS 2016, 241 (2016)

[16] D. Boer, C. Lorce, C. Pisano, and J. Zhou, Adv. High Energy Phys. 2015, 760840 (2015)

[17] A. Mocsy, Eur. Phys. J. C 61, 705 (2009)

[18] I. Karpenko, arXiv: 1805.11998 [nucl-th]. 
[19] F. Becattini and J. Cleymans, J. Phys. G 34, S959 (2007)

[20] V. Begun, D. Kikola, V. Vovchenko, and D. Wielanek, arXiv: 1806.01303 [nucl-th]

[21] B. Trzeciak, C. Da Silva, E. Ferreiro et al., Few-Body Syst. 58, 148 (2017)

[22] A. B. Kurepin and N. S. Topilskaya, Adv. High Energy Phys. 2015, 760840 (2015)

[23] R. Aaij et al. (LHCb Collaboration), JINST 9, P12005 (2014)

[24] A. Airapetian et al. (HERMES Collaboration), Nucl. Instrum. Meth. A 540, 68 (2005)

[25] A. Zelenski et al., Nucl. Instrum. Meth. A 536, 248 (2005)

[26] C. Barschel, P. Lenisa, A, Nass, and E. Steffens, Adv. High Energy Phys. 2015, 463141 (2015)

[27] T. Pierog, I. Karpenko, J. M. Katzy, E. Yatsenko, and K. Werner, Phys. Rev. C 92, 034906 (2015)

[28] J. K. Werner, F.-M. Liu, and T. Pierog, Phys. Rev. C 74, 044902 (2006) 